TITLE

Identification and Characterization of Sulfur Heterocyclic Compounds That Contribute to the Acidic Odor of Aged Garlic Extract

\title{
AUTHORSHIP
}

Kazuki Abe, ${ }^{\dagger, \dagger}$ Takao Myoda, ${ }^{\dagger}$ and Satoshi Nojima* ${ }^{*} \dagger$

†Laboratory of Aroma Chemistry, Department of Food and Cosmetic Science, Faculty of Bioindustry, Tokyo University of Agriculture, 196 Yasaka Abashiri City Hokkaido 099-2493, Japan

Healthcare Research and Development Division, Wakunaga Pharmaceutical Co. Ltd., 1624 Shimokotachi, Kodacho, Akitakata, Hiroshima 739-1195, Japan

Telephone number of correspondent: $+81-152-48-3820$

E-mail address of correspondent: sn207003@nodai.ac.jp 
Figure S1

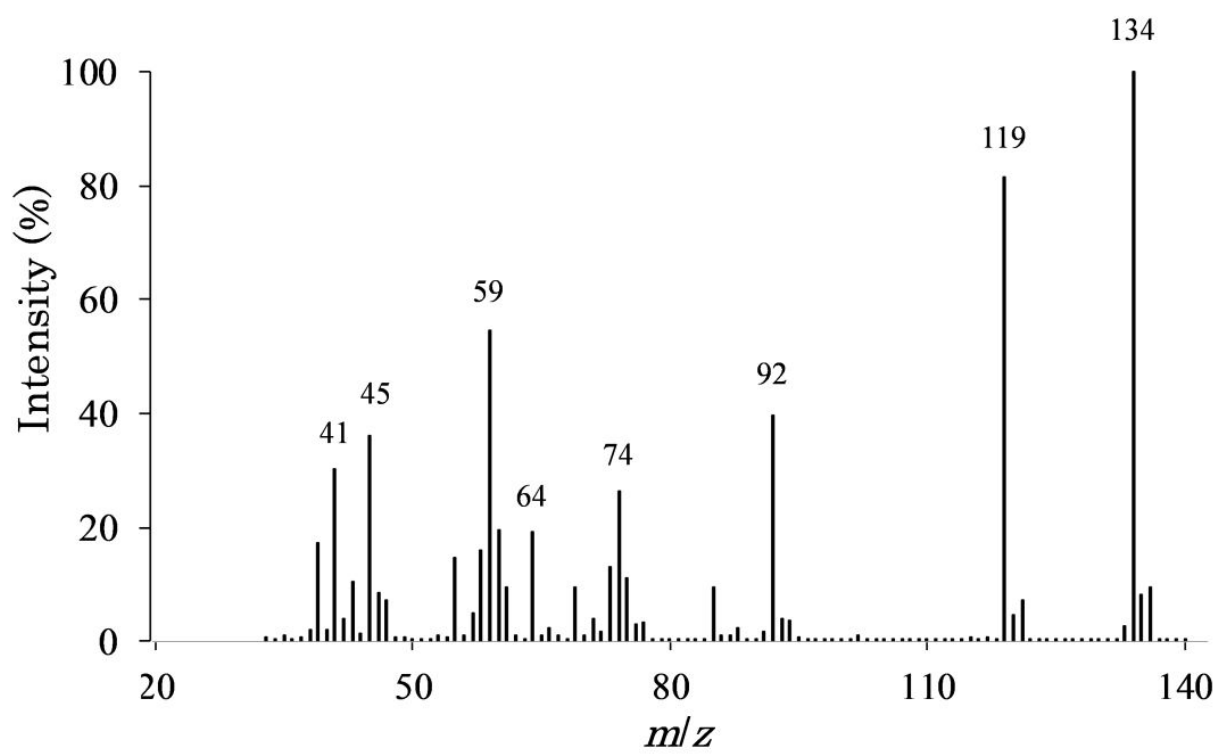

Figure S1. Mass spectrum of cis-2,4-dimethyl-1,3-dithiolane in AGE. 


\section{Figure S2}

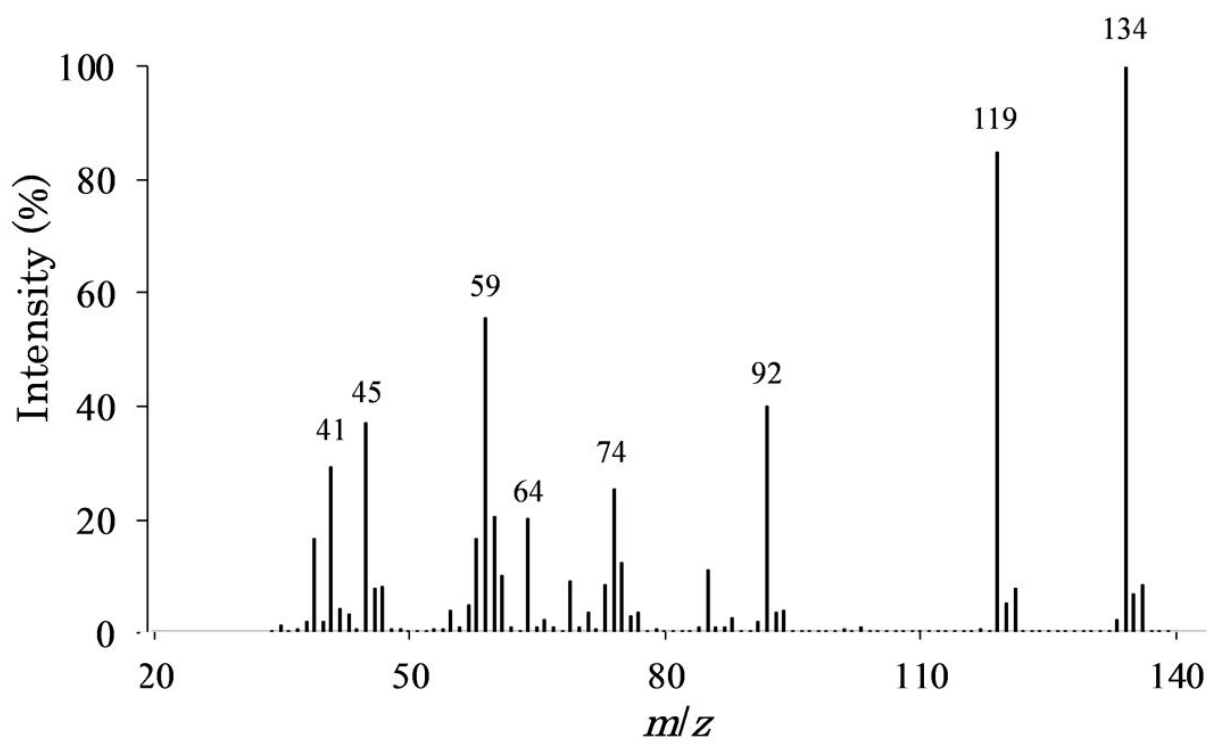

Figure S2. Mass spectrum of trans-2,4-dimethyl-1,3-dithiolane in AGE. 
Figure S3

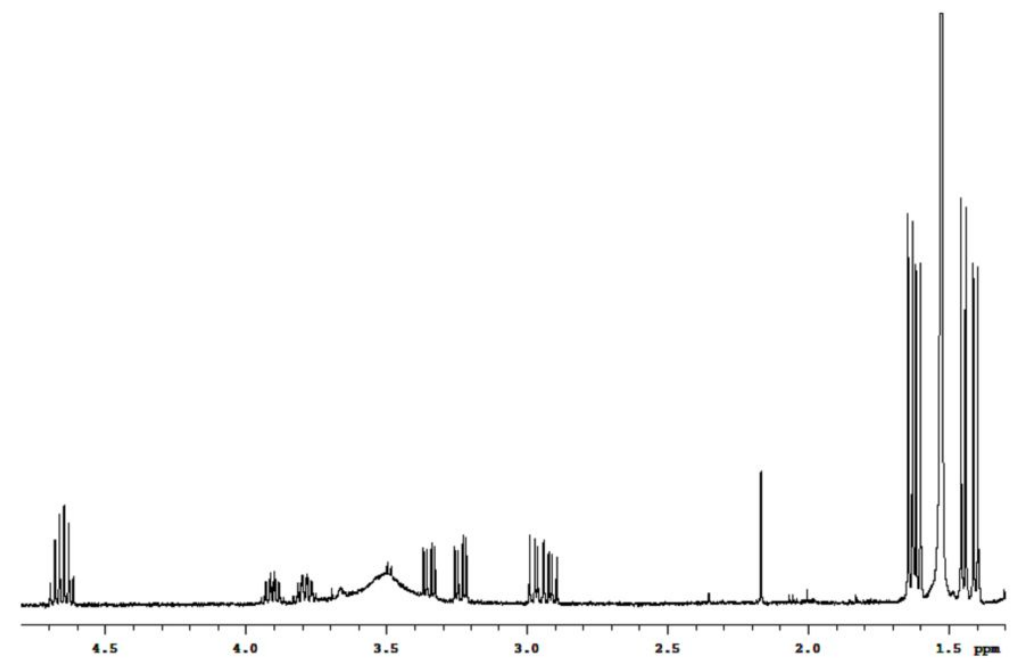

Figure S3. ${ }^{1} \mathrm{H}-\mathrm{NMR}$ spectrum of the mixture of cis-2,4-dimethyl-1,3-dithiolane and trans-2,4-dimethyl-1,3-dithiolane in AGE. 


\section{Figure S4}

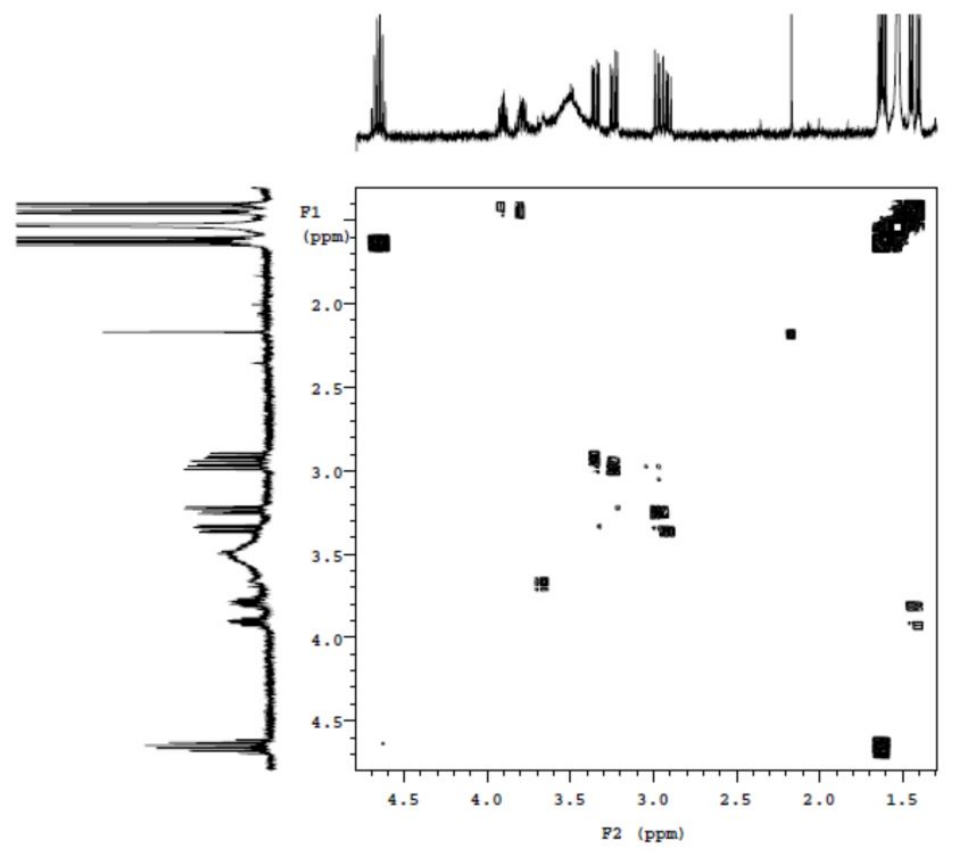

Figure S4. ${ }^{1} \mathrm{H}-{ }^{1} \mathrm{H}$ COSY spectrum of the isolated mixture of cis-2,4-dimethyl-1,3-dithiolane and trans-2,4-dimethyl-1,3-dithiolane in AGE. 
Figure S5

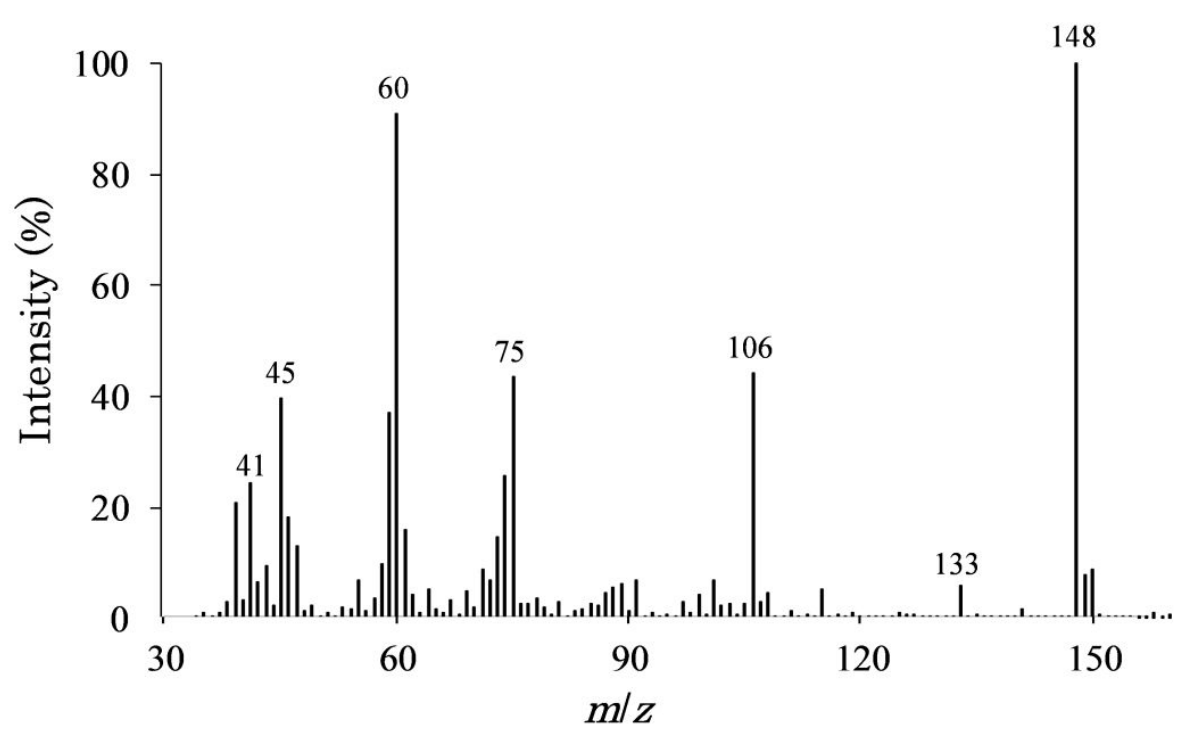

Figure S5. Mass spectrum of cis-2,5-dimethyl-1,4-dithiane in AGE. 
Figure S6

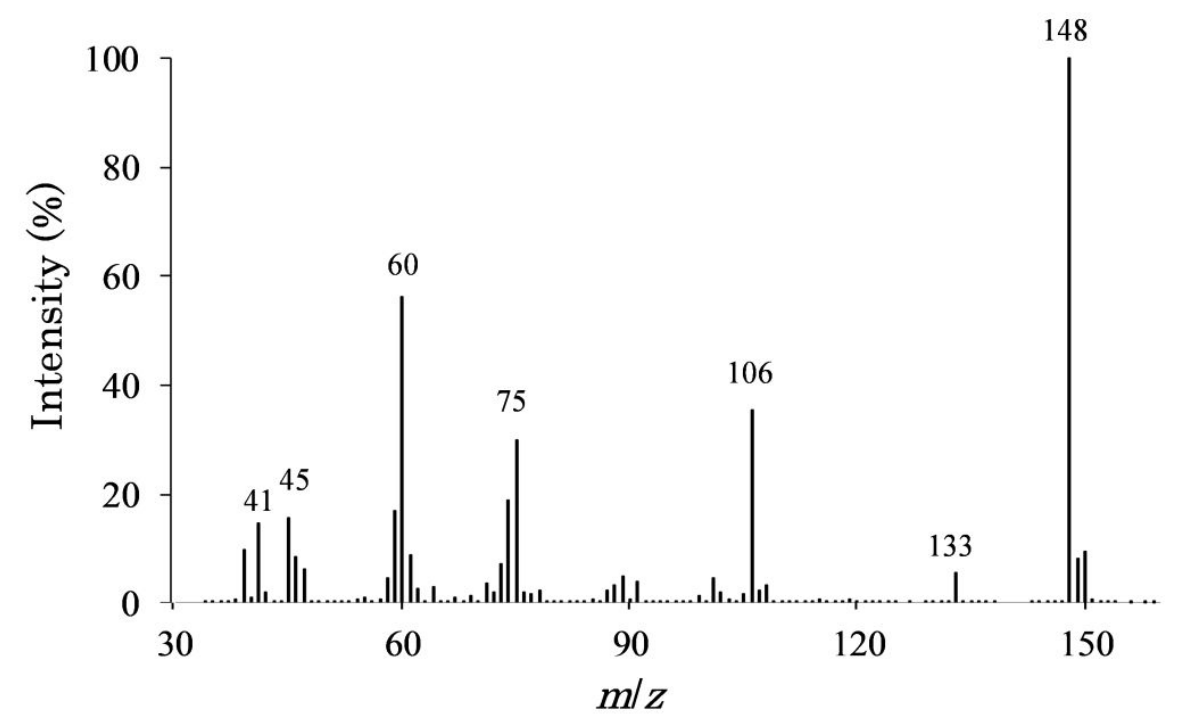

Figure S6. Mass spectrum of trans-2,5-dimethyl-1,4-dithiane in AGE. 
Figure S7

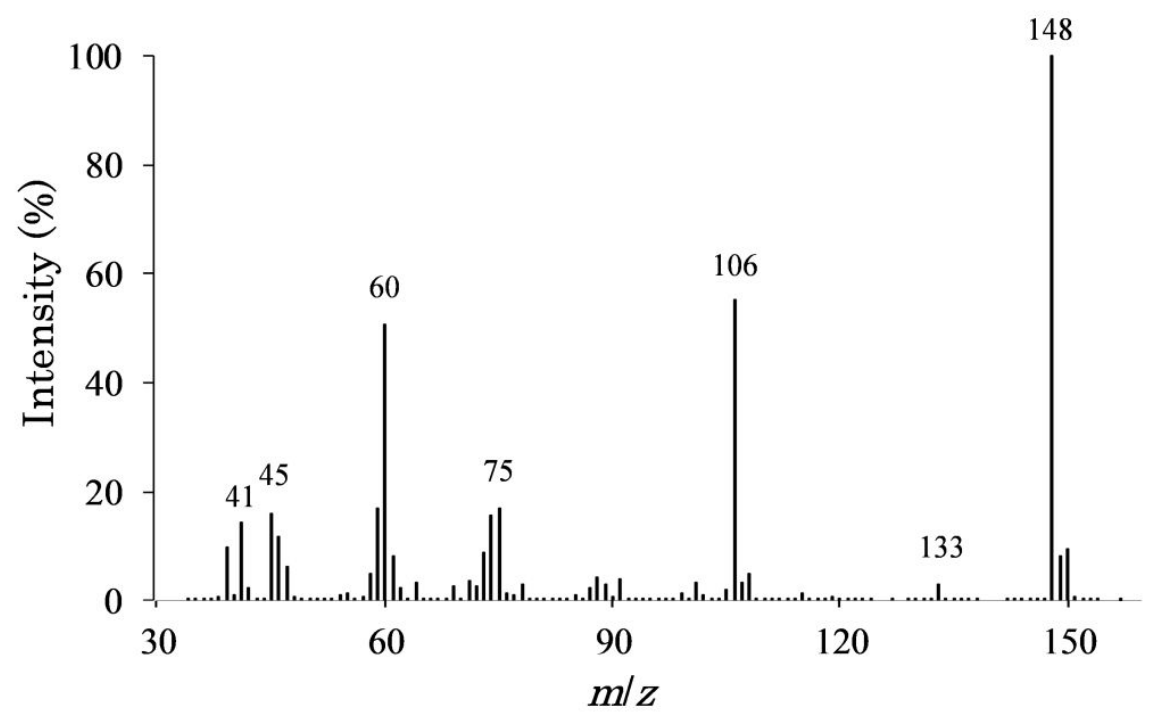

Figure S7. Mass spectrum of cis-2,6-dimethyl-1,4-dithiane in AGE. 
Figure S8

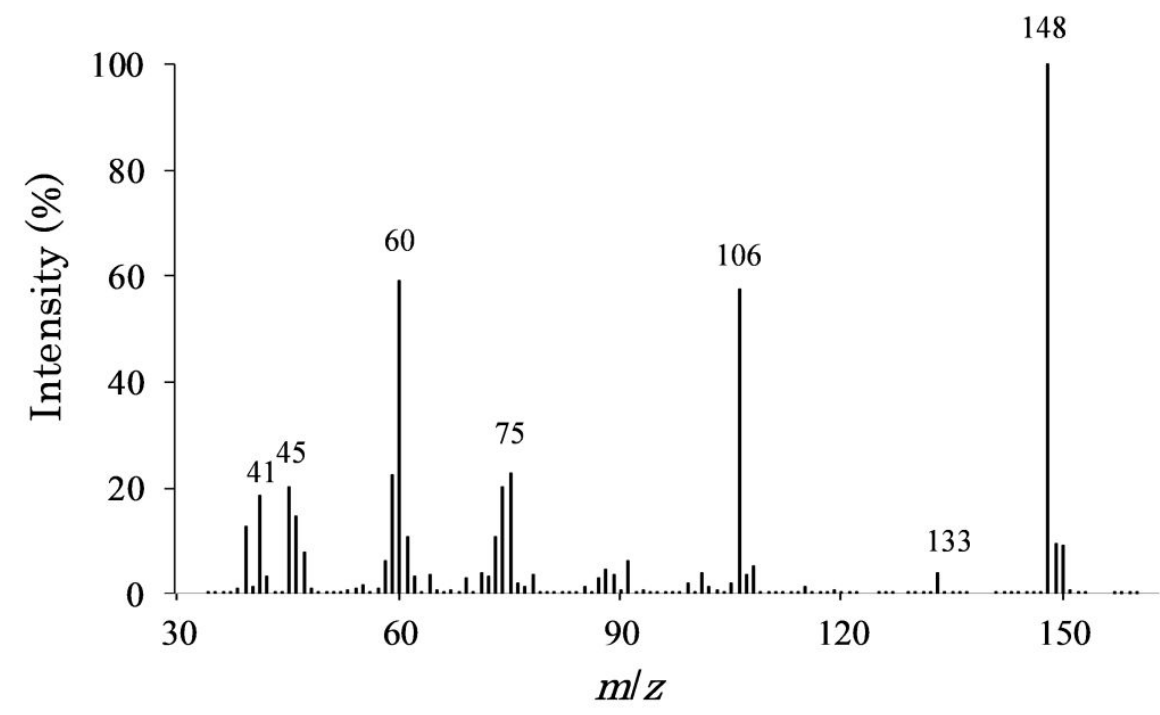

Figure S8. Mass spectrum of trans-2,6-dimethyl-1,4-dithiane in AGE. 


\section{Figure S9}

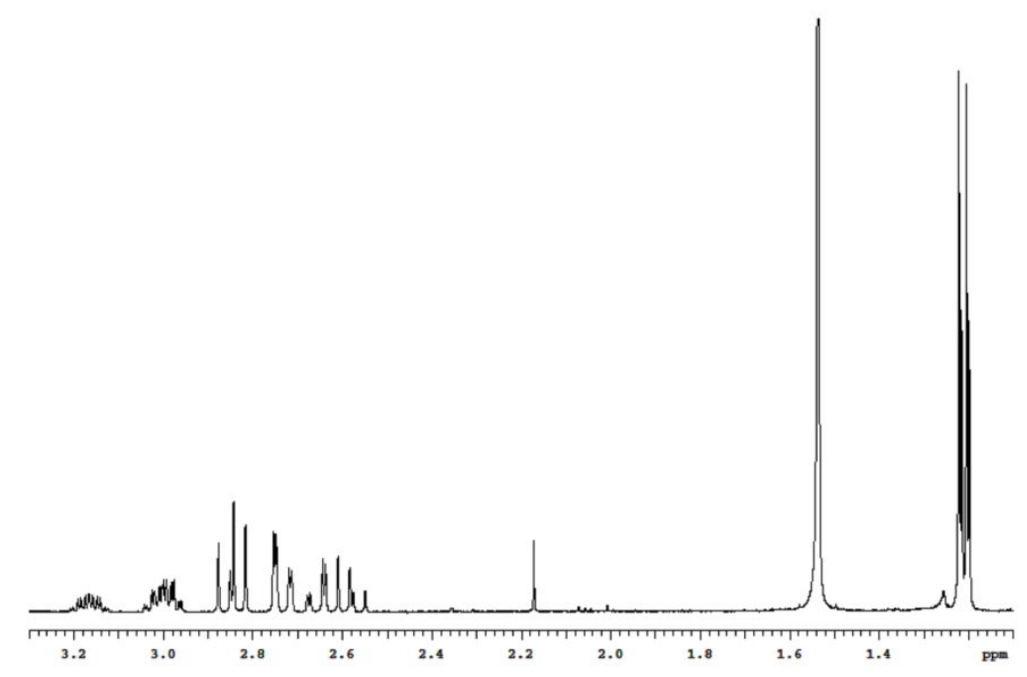

Figure S9. ${ }^{1} \mathrm{H}-\mathrm{NMR}$ spectrum of the mixture of trans-2,5-dimethyl-1,4-dithiane and cis-2,6-dimethyl-1,4-dithiane in AGE. 
Figure S10

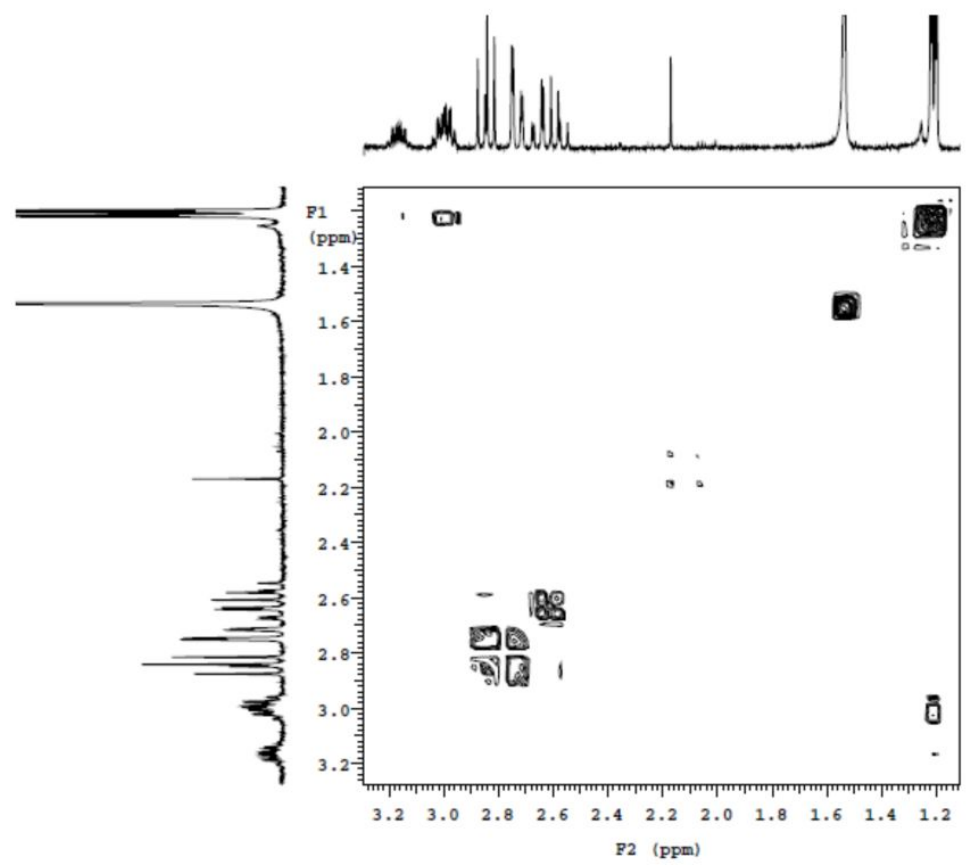

Figure S10. ${ }^{1} \mathrm{H}-{ }^{1} \mathrm{H}$ COSY spectrum of the isolated mixture of trans-2,5-dimethyl-1,4-dithiane and cis-2,6-dimethyl-1,4-dithiane in AGE. 


\section{Figure S11}

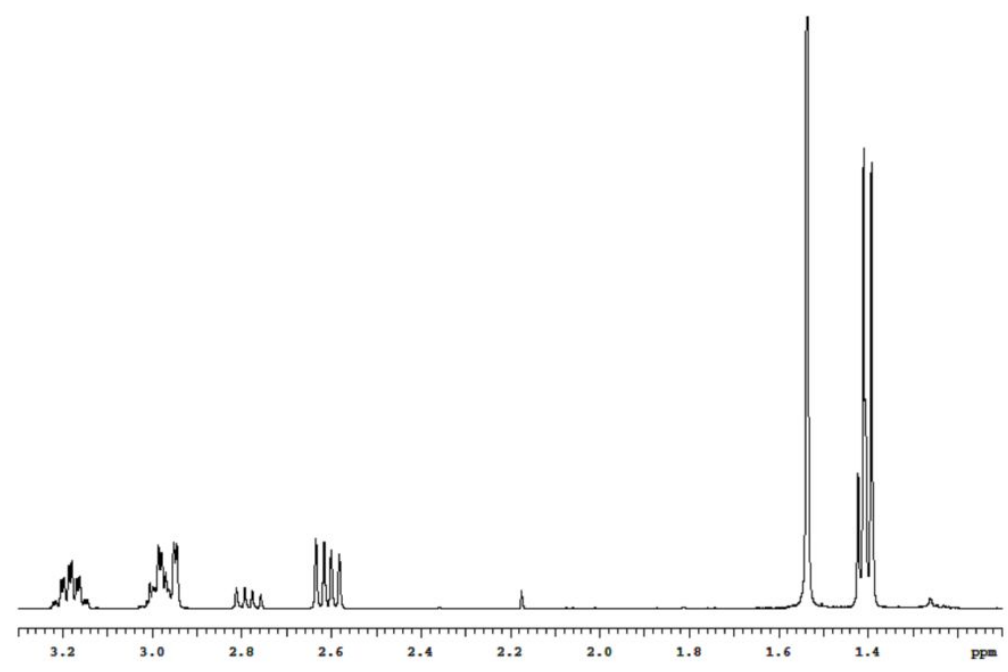

Figure S11. ${ }^{1} \mathrm{H}-\mathrm{NMR}$ spectrum of the mixture of cis-2,5-dimethyl-1,4-dithiane and trans-2,6-dimethyl-1,4-dithiane in AGE. 


\section{Figure S12}

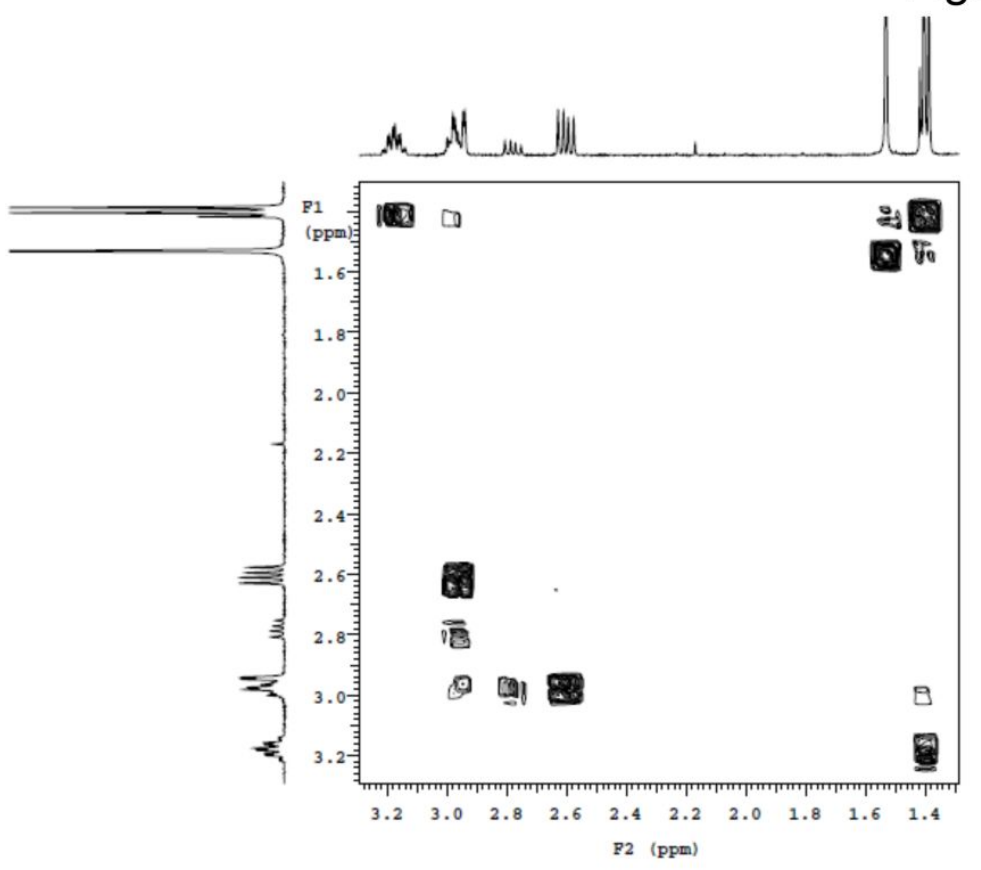

Figure S12. ${ }^{1} \mathrm{H}-{ }^{1} \mathrm{H}$ COSY spectrum of the isolated mixture of $c$ is-2,5-dimethyl-1,4-dithiane and trans-2,6-dimethyl-1,4-dithiane in AGE. 